\title{
Development of expressed sequence tag resources for Vanda Mimi Palmer and data mining for EST-SSR
}

\begin{abstract}
Vanda Mimi Palmer (VMP) is a highly sought as fragrant orchid hybrid in Malaysia. It is economically important in cosmetic and beauty industries and also a famous potted ornamental plant. To date, no work on fragrance-related genes of vandaceous orchids has been reported from other research groups although the analysis of floral fragrance or volatiles have been extensively studied. An expressed sequence tag (EST) resource was developed for VMP principally to mine any potential fragrance-related expressed sequence tag-simple sequence repeat (EST-SSR) for future development as markers in the identification of fragrant vandaceous orchids endemic to Malaysia. Clustering, annotation and assembling of the ESTs identified 1,196 unigenes which defined 966 singletons and 230 contigs. The VMP dbEST was functionally classified by gene ontology (GO) into three groups:molecular functions $(51.2 \%)$, cellular components $(16.4 \%)$ and biological processes $(24.6 \%)$ while the remaining $7.8 \%$ showed no hits with GO identifier. A total of 112 EST-SSR(9.4\%) was mined on which at least five units of di-, tri-,tetra-, penta-, or hexa-nucleotide repeats were predicted.The di-nucleotide motif repeats appeared to be the most frequent repeats among the detected SSRs with the AT/TA types as the most abundant among the dimerics, while AAG/TTC, AGA/TCT-type were the most frequent trimerics. The mined EST-SSR is believed to be useful in the development of EST-SSR markers that is applicable in the screening and characterization of fragrance-related transcripts in closely related species.
\end{abstract}

Keyword: Expressed sequence tag; Orchids; Simple sequence repeat; Vanda Mimi Palmer. 